\title{
Dynamics of translational and rotational thermalization of AlF molecules via collisions with cryogenic helium
}

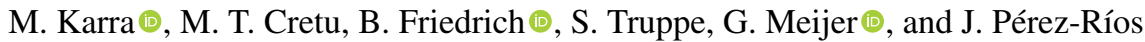 \\ Fritz-Haber-Institut der Max-Planck-Gesellschaft, Faradayweg 4-6, 14195 Berlin, Germany
}

(Received 12 October 2021; accepted 7 January 2022; published 11 February 2022)

\begin{abstract}
We investigated helium-mediated translational and rotational thermalization of the aluminum monofluoride (AlF) molecule at cryogenic temperatures via an ab initio potential energy surface (PES) and quantum multichannel scattering theory. Our examination of the elastic and rotationally inelastic channels revealed that helium is an efficient quencher of $\mathrm{AlF}$ at temperatures relevant to buffer gas cooling experiments ( $\sim 1 \mathrm{mK}$ to $10 \mathrm{~K})$. We also showed that this conclusion is robust against possible inaccuracies of the PES.
\end{abstract}

DOI: 10.1103/PhysRevA.105.022808

\section{INTRODUCTION}

The ability to cool gaseous ensembles of molecules to cold $(1 \mathrm{mK}$ to $10 \mathrm{~K})$ and ultracold $(\lesssim 1 \mathrm{mK})$ temperatures has been impacting research areas as diverse as testing the standard model of particle physics [1], quantum-logic spectroscopy [2-6], action spectroscopy [7-9], chemical reaction dynamics [10-16], as well as quantum computing [17-19] and quantum simulation [20,21]. In particular, ultracold polar molecules afford tunability of interactions, coveted for applications in quantum information protocols $[22,23]$ and the simulation of many-body Hamiltonians [24,25].

Ultracold molecules can be produced via direct or indirect cooling techniques. The indirect techniques rely on photoassociation [26-30] or magnetoassociation [31,32] of a pair of ultracold atoms and have proved especially successful for generating bialkali ultracold molecules [33-38]. The yield of the indirect techniques is limited by the number of available ultracold atoms that serve as precursors of the ultracold molecules. However, bialkali molecules are prone to undergoing sticky collisions: these correspond to the formation of an intermediate complex whose lifetime can be long enough to amount to a loss of the molecules that are being formed [39-41].

On the other hand, direct cooling techniques are based on the dissipation of translational-and internal-energy of preexisting molecules via thermalization with a cryogenic buffer gas [42], Stark [43] or Zeeman [44,45] slowing of molecules cooled by a supersonic expansion, and Sisyphus cooling [46] or, most recently, laser cooling [47-49]. Long considered impractical if not impossible [10], laser cooling of molecules has led, for the first time, to the creation

Published by the American Physical Society under the terms of the Creative Commons Attribution 4.0 International license. Further distribution of this work must maintain attribution to the author(s) and the published article's title, journal citation, and DOI. Open access publication funded by the Max Planck Society. of ultracold molecular ensembles [50-52]. As laser cooling of molecules requires near-unity Franck-Condon factors between the molecular ground and excited electronic states [47,53], only a handful of molecules have been laser cooled so far [52,54-56].

Recently, we identified the aluminum monofluoride (AlF) molecule as a promising candidate for laser cooling. Its virtues include a large photon scattering rate and favorable Franck-Condon factors [57-59]. Moreover, we demonstrated that AlF molecules can be efficiently produced in a pulsed buffer-gas source, with a yield of $\gtrsim 10^{12}$ molecules per steradian per pulse [58]. A high yield of cryogenically thermalized molecules is a key prerequisite for reaching quantum degeneracy in a cooling step that follows upon laser cooling, namely, forced evaporation.

In order to gain insight into the thermalization of $\mathrm{AlF}$ by cryogenic helium, we undertook a study of the quantum dynamics of the elastic and rotationally inelastic scattering of $\mathrm{AlF}$ by $\mathrm{He}$ at collision energies ranging from $1 \mathrm{mK}$ to $10 \mathrm{~K}$. We made use of an accurate $a b$ initio potential energy surface (PES) and calculated the relevant scattering observables via the coupled-channel method. The hyperfine structure of AlF has been omitted as it is expected to play only a minor role in the collision dynamics. We also discuss what our findings about the AlF-He system imply about the thermalization in a buffer gas of other $\Sigma$ diatomic fluoride candidates for laser cooling, such as $\mathrm{MgF}$ and $\mathrm{BaF}$.

We note that following the discovery of its presence in the envelope of protoplanetary nebulae [60], the AlF molecule and its spectroscopic properties have been also of astronomical interest. More recently, the $\gamma$-ray emission from ${ }^{26} \mathrm{AlF}$ has served as a stepping stone to elucidating the Galactic sources of ${ }^{26} \mathrm{Al}$ [61], which is of consequence for understanding the frequency of core collapse of supernovae [62].

\section{POTENTIAL ENERGY SURFACE}

The potential energy surface of the ${ }^{27} \mathrm{Al}^{19} \mathrm{~F}\left(X^{1} \Sigma^{+}\right)+{ }^{4} \mathrm{He}(1 S)$ system was calculated by treating $\operatorname{AlF}\left(X^{1} \Sigma^{+}\right)$like a vibrationless rigid rotor. Thus, the 


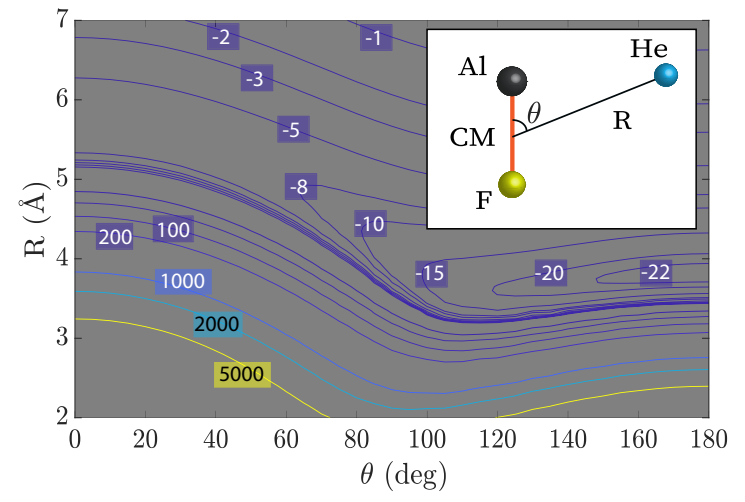

FIG. 1. Potential energy surface (PES) of the $\operatorname{AlF}\left(X^{1} \Sigma^{+}\right)-$ $\mathrm{He}\left({ }^{1} S\right)$ system. The contours show fixed electronic energies in $\mathrm{cm}^{-1}$ of the system as a function of $R$ and $\theta$, whose meaning is defined in the inset. See, also, the text.

PES only depends on two parameters: the distance $R=|\mathbf{R}|$ between the center of mass of the molecule and the atom, and the angle $\theta$ between $\mathbf{R}$ and the internuclear axis $\mathbf{r}$ of the AlF molecule; see the inset in Fig. 1. The PES was calculated at the spin-restricted open-shell coupled-cluster level of theory $(\operatorname{RCCSD}(\mathrm{T}))$ as implemented in MOLPRO [63], including single and double excitations with the triples excitations being treated perturbatively. We used the standard aug-cc-pv5Z basis set of Dunning [64]. The Al-F equilibrium distance was fixed to that accurately determined in a recent experiment $[57,65], R_{e}=1.654369 \AA$. The basis set superpostion error was corrected using the counterpoise method. A contour plot of the PES is shown in Fig. 1. Note that the AlF-He attraction is quite weak, as it arises from the van der Waals interaction between a closed-shell atom and a closed-shell molecule. The PES exhibits a global minimum of $\sim 22 \mathrm{~cm}^{-1}$ close to the $\mathrm{F}$ atom, lending it a high anisotropy characteristic of fluorine-containing heteronuclear molecules interacting with $\mathrm{He}$ [66].

The PES was calculated for 1500 geometries including 20 angles, 18 of which corresponded to the Gauss-Legendre quadrature points and to $\theta=0$ and $\theta=\pi$, and 75 radial points between 1.6 and $30 \AA$ with a larger density of points for $R$ between 3 and $5 \AA$. The global minimum of the PES was found at $\theta=\pi$ and $R=3.8 \AA$, in close agreement with previously reported calculations [67]. The technical details of calculating the PES will be published elsewhere.

For scattering calculations, it is advantageous to expand the PES in a Legendre series,

$$
V(R, \theta)=\sum_{\lambda=0}^{\lambda_{\max }} V_{\lambda}(R) P_{\lambda}(\cos \theta),
$$

with $P_{\lambda}(\cos \theta)$ Legendre polynomials of degree $\lambda$ and expansion coefficients

$$
V_{\lambda}(R)=\left(\frac{2 \lambda+1}{2}\right) \int_{0}^{\pi} V(R, \theta) P_{\lambda}(\cos \theta) \sin \theta d \theta,
$$

which depend solely on the radial distance $R$. Note that $V_{0}(R)$ represents the only isotropic (spherical) contribution to the PES, whereas all other contributions are anisotropic. The integral in Eq. (2) was performed using the Gauss-Legendre

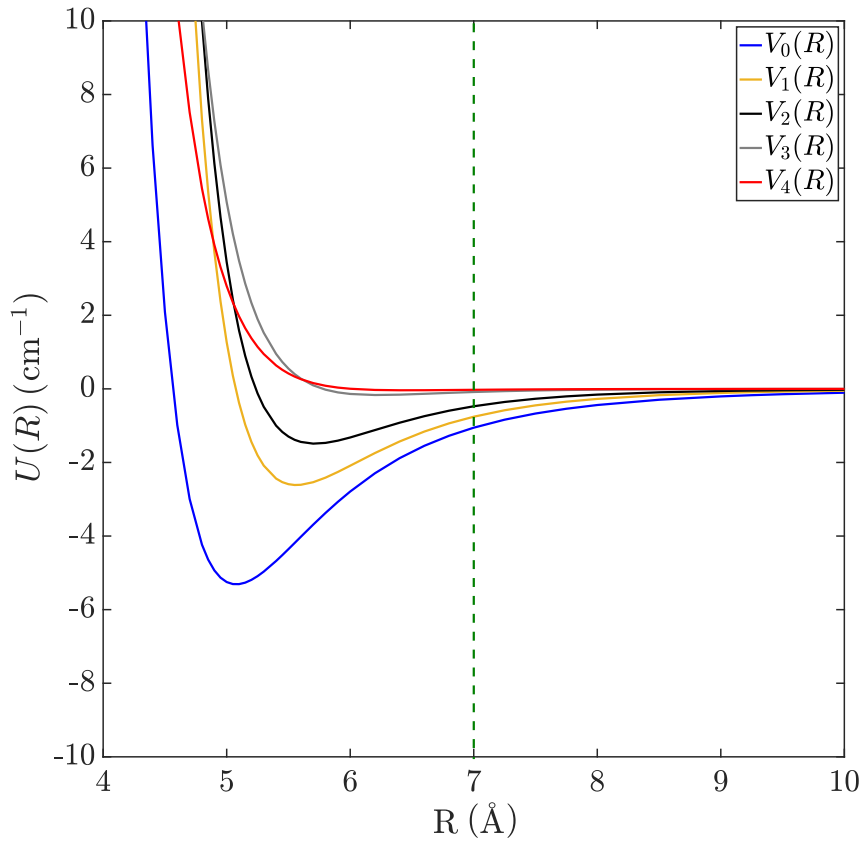

FIG. 2. The first five radial terms $V_{\lambda}(R)$ of the potential energy surface of the $\operatorname{AlF}\left(X^{1} \Sigma^{+}\right)-\mathrm{He}(1 S)$ system; see text.

quadrature scheme employing 18 quadrature points. The dependence of the first five $V_{\lambda}(R)$ terms on $R$ is shown in Fig. 2. As expected, the most significant radial term is the spherical one, $V_{0}(R)$. By including radial terms with up to $\lambda_{\max }=12$ in the Legendre series expansion of Eq. (1), we ensured a relative error of the Legendre expansion of only $0.1 \%$ with respect to the $a b$ initio PES.

For cold and ultracold collisions, the long-range tail of the PES plays a critical role for evaluating the scattering cross sections. Therefore, we chose a certain $R_{\text {mid }}$ for each $V_{\lambda}$ beyond which the radial coefficient was replaced by the long-range dipole-induced dipole interaction,

$$
V_{\lambda}\left(R \geqslant R_{\text {mid }}\right)=\frac{C_{6}^{\lambda}}{R^{6}} .
$$

The values of $C_{6}^{\lambda}$ were obtained by fitting the numerical values from Eq. (2) between $R_{\text {mid }}$ and $30 \AA$. Additionally, a polynomial interpolation was used to ensure the continuity of the potential and its first derivative at $R_{\text {mid }}$.

\section{COLLISION DYNAMICS}

In evaluating the quantum dynamics of the $\mathrm{AlF}+\mathrm{He}$ collisions, we make use of the framework developed by Arthurs et al. [68] for rigid rotor-atom collisions, omitting the hyperfine structure.

\section{A. State-to-state integral cross sections}

The state-to-state integral scattering cross section for collisions leading from an initial state $j$ of the rotor to its final state $j^{\prime}$ is given by

$$
\sigma_{j \rightarrow j^{\prime}}\left(E_{k}\right)=\frac{4 \pi}{k^{2}(2 j+1)} \sum_{J l l^{\prime}}(2 J+1)\left|T_{j, l ; j^{\prime} l^{\prime}}^{J M}\right|^{2},
$$


where $E_{k}$ is the collision energy, $k$ the wave number of the relative atom-molecule motion, $k^{2}=2 \mu E_{k}$, with $\mu$ the reduced mass of the atom-molecule system, and $T_{j, l ; j^{\prime} l^{\prime}}^{J M}$ is the transition matrix that encapsulates the transition probabilities between different scattering channels characterized by the rotational quantum numbers $j$ and $j^{\prime}$ and partial waves $l$ and $l^{\prime}$ before and after the collision. In the absence of external fields, only those channels $(j, l)$ and $\left(j^{\prime}, l^{\prime}\right)$ are coupled that conserve the total angular momentum $J$ and its projection $M$ on the quantization axis (scattering coordinate $\mathbf{R}$ ). Note that throughout this section, we use atomic units.

In the center-of-mass frame, the Hamiltonian of the atommolecule system is given by

$$
H=-\frac{1}{2 \mu} \nabla_{R}^{2}+\frac{\hat{l}^{2}}{2 \mu R^{2}}+V(R, \theta)+H_{\text {rot }},
$$

where the first term represents the radial kinetic energy operator along the scattering coordinate $\mathbf{R}$, the second term denotes the centrifugal term of the kinetic energy operator, $V(R, \theta)$ the atom-molecule PES (cf. Sec. II), and $H_{\text {rot }}$ the rotational Hamiltonian of the molecule. The last is given by

$$
H_{\mathrm{rot}} Y_{j}^{m_{j}}(\hat{r})=\left[B_{0} j(j+1)-D_{j}(j(j+1))^{2}\right] Y_{j}^{m_{j}}(\hat{r}),
$$

with $Y_{j}^{m_{j}}(\hat{r})$ the spherical harmonics, $B_{0}$ the rotational constant of the vibrational ground state of the molecule, $D_{j}$ the centrifugal distortion constant, $j$ the rotational quantum number, and $m_{j}$ its projection on the direction of the molecular axis, $\hat{r}=\frac{\mathbf{r}}{|\mathbf{r}|}$.

The total energy of the rotor is comprised of its kinetic energy and the internal energy of the fragment [69] it corresponds to:

$$
E_{j}=E_{k}+B_{0} j(j+1)-D_{j}[j(j+1)]^{2},
$$

which is an eigenvalue of the Schrödinger equation

$$
H \Psi_{j l}^{J M}=E_{j} \Psi_{j l}^{J M} .
$$

In the coupled basis, preferable in the absence of external fields, the eigenfunctions of Eq. (8) are given by

$$
\Psi_{j l}^{J M}=\sum_{j^{\prime} l^{\prime}} \frac{g_{j l, j^{\prime} l^{\prime}}^{J M}(R)}{R} I_{j^{\prime} l^{\prime}}^{J M}(\hat{r}, \hat{R}),
$$

with

$$
I_{j l}^{J M}(\hat{r}, \hat{R})=\sum_{m_{j} m_{l}} C_{j m_{j} l m_{l}}^{J M}\left|j m_{j} l m_{l}\right\rangle
$$

the angular part of the wave function that entails the coupling of the molecule-fixed coordinate $\hat{r}$ to the scattering coordinate $\hat{R}=\frac{\mathbf{R}}{|\mathbf{R}|}$ via the Clebsch-Gordan coefficients $C_{j m_{j} l m_{l}}^{J M}$.

By substituting Eq. (9) into Eq. (8), we obtain

$$
\begin{aligned}
& {\left[-\frac{d^{2}}{d R^{2}}+k_{j^{\prime}}^{2}+\frac{l^{\prime}\left(l^{\prime}+1\right)}{R^{2}}\right] g_{j l, j^{\prime} l^{\prime}}^{J M}(R)} \\
& =2 \mu \sum_{j^{\prime \prime} l^{\prime \prime}} V_{j^{\prime \prime} l^{\prime \prime} ; j^{\prime} l^{\prime}}^{J}(R) g_{j l, j^{\prime \prime} l^{\prime \prime}}^{J M}(R),
\end{aligned}
$$

where

$$
\begin{aligned}
k_{j^{\prime}}^{2}= & 2 \mu\left\{E_{k}+B_{0} j(j+1)-D_{j}[j(j+1)]^{2}\right. \\
& \left.-B_{0} j^{\prime}\left(j^{\prime}+1\right)+D_{j}\left[j^{\prime}\left(j^{\prime}+1\right)\right]^{2}\right\}
\end{aligned}
$$

is the wave number squared pertaining to the $\left(j, j^{\prime}\right)$ channel, and

$$
V_{j^{\prime} l^{\prime} ; j l}^{J}(R)=\iint I_{j^{\prime} l^{\prime}}^{J M *}(\hat{r}, \hat{R}) V(R, \theta) I_{j l}^{J M}(\hat{r}, \hat{R}) d \Omega_{r}
$$

are the potential matrix elements. By substituting from Eq. (1), we obtain

$$
\begin{aligned}
V_{j^{\prime} l^{\prime} ; j l}^{J}(R)= & \sum_{\lambda}^{\lambda_{\max }} V_{\lambda}(R)(-1)^{J+l^{\prime}+l} \\
& \times \sqrt{(2 j+1)(2 l+1)\left(2 j^{\prime}+1\right)\left(2 l^{\prime}+1\right)} \\
& \times\left(\begin{array}{ccc}
l & \lambda & l^{\prime} \\
0 & 0 & 0
\end{array}\right)\left(\begin{array}{ccc}
j & \lambda & j^{\prime} \\
0 & 0 & 0
\end{array}\right)\left\{\begin{array}{ccc}
j & \lambda & j^{\prime} \\
l^{\prime} & J & l
\end{array}\right\},
\end{aligned}
$$

with $(\cdot)$ and $\{\cdot\}$ the $3 j$ and $6 j$ symbols, respectively. Thus the simple form of Eq. (14) results by virtue of the Legendre expansion of the PES, introduced in Sec. II.

The set of coupled differential equations (11) is solved numerically. In the asymptotic region where the interaction potential is negligible compared to the collision energy, the numerical results can be matched to the expected analytic asymptotic behavior,

$$
\begin{array}{r}
g_{j l, j^{\prime} l^{\prime}}^{J M}(R \rightarrow \infty) \sim \delta_{j j^{\prime}} \delta_{l l^{\prime}} \sin \left(k_{j^{\prime}} R-\frac{l^{\prime} \pi}{2}\right) \\
+\frac{e^{l\left(k_{j^{\prime}} R-l^{\prime} \pi / 2\right)}}{\sqrt{k_{j^{\prime}}}} T_{j l, j^{\prime} l^{\prime}}^{J M},
\end{array}
$$

leading to the numerical transition matrix and thus, via Eq. (4), to the state-to-state cross section.

\section{B. Diffusion and viscosity transport cross sections}

The diffusion and viscosity cross sections are defined by [70]

$$
\sigma_{D}\left(E_{k}\right)=\int \frac{d \sigma_{\mathrm{el}}\left(E_{k}\right)}{d \Omega}(1-\cos \theta) d \Omega
$$

and

$$
\sigma_{\eta}\left(E_{k}\right)=\int \frac{d \sigma_{\mathrm{el}}\left(E_{k}\right)}{d \Omega}\left(1-\cos ^{2} \theta\right) d \Omega,
$$

where $\frac{d \sigma_{\mathrm{el}}\left(E_{k}\right)}{d \Omega}$ is the elastic differential cross section, $d \Omega=$ $2 \pi \sin \theta d \theta$ the solid angle element, and $\theta$ the scattering angle. Quantum mechanically, these cross sections evaluate to [70]

$$
\sigma_{D}\left(E_{k}\right)=\frac{4 \pi}{k^{2}} \sum_{l=0}^{\infty}(l+1) \sin ^{2}\left[\delta_{l+1}\left(E_{k}\right)-\delta_{l}\left(E_{k}\right)\right]
$$

and

$$
\sigma_{\eta}\left(E_{k}\right)=\frac{4 \pi}{k^{2}} \sum_{l=0}^{\infty} \frac{(l+1)(l+2)}{2 l+3} \sin ^{2}\left[\delta_{l+2}\left(E_{k}\right)-\delta_{l}\left(E_{k}\right)\right],
$$

respectively, wherein $\delta_{l}\left(E_{k}\right)$ is the phase shift for a given partial wave $l$ and collision energy $E_{k}$. The quantum elastic 
scattering cross section expressed in terms of the phase shifts boils down to

$$
\sigma_{\mathrm{el}}\left(E_{k}\right)=\frac{4 \pi}{k^{2}} \sum_{l=0}^{\infty}(2 l+1) \sin ^{2} \delta_{l} .
$$

Classically, the transport cross sections are given by $[71,72]$

$$
\begin{array}{r}
\sigma_{D}^{\mathrm{cl}}\left(E_{k}\right)=2 \pi \int_{0}^{\infty}\left\{1-\cos \left[\chi\left(E_{k}, b\right)\right]\right\} b d b, \\
\sigma_{\eta}^{\mathrm{cl}}\left(E_{k}\right)=2 \pi \int_{0}^{\infty}\left\{1-\cos ^{2}\left[\chi\left(E_{k}, b\right)\right]\right\} b d b,
\end{array}
$$

with $\chi$ the deflection angle and $b$ the impact parameter:

$$
\chi\left(E_{k}, b\right)=\pi-2 b \int_{R_{c}}^{\infty} \frac{R^{-2} d R}{\sqrt{1-V_{0}(R) / E_{k}-b^{2} / R^{2}}},
$$

where $R_{c}$ is the distance of closest approach in the collision, obtained by solving

$$
1-V_{0}\left(R_{c}\right) / E_{k}-b^{2} / R_{c}^{2}=0 .
$$

Integration of $\chi$ over all impact parameters then yields $\sigma_{D}$ and $\sigma_{\eta}$, as given above by Eqs. (21) and (22).

The expressions for the transport cross sections can be thermally averaged, resulting in

$$
\sigma_{D}^{\mathrm{cl}}(T)=\frac{1}{2\left(k_{B} T\right)^{3}} \int_{0}^{\infty} \sigma_{D}\left(E_{k}\right) \exp \left[-\frac{E_{k}}{k_{B} T}\right] E_{k}^{2} d E_{k}
$$

and

$$
\sigma_{\eta}^{\mathrm{cl}}(T)=\frac{1}{6\left(k_{B} T\right)^{4}} \int \sigma_{\eta}\left(E_{k}\right) \exp \left[-\frac{E_{k}}{k_{B} T}\right] E_{k}^{3} d E_{k},
$$

with $k_{B}$ the Boltzmann constant and $T$ the temperature. These thermally averaged cross sections occur in the kinetic theory of gas transport [72].

\section{COMPUTATIONAL DETAILS}

\section{A. Classical cross sections}

The classical cross sections were calculated by solving Eqs. (21) to (24) numerically using PYTHON. Only the spherical component of the PES, $V_{0}(R)$, was taken into account; cf. Eqs. (23) and (24).

\section{B. Quantum elastic cross sections}

The quantum elastic cross sections were obtained by solving the single-channel Schrödinger equation using Numerov's method. We employed $10^{5}$ steps between $3.6 a_{0}$ and $R_{\max }$ (300 $a_{0}$ for the lowest collision energy and $60 a_{0}$ for the highest) including an appropriate number of partial waves to guarantee a convergence better than $1 \%$. And, as in the case of the classical calculations, only the spherical component $V_{0}(R)$ of the potential was used.

\section{Quantum multichannel calculations}

Throughout this study, we made use of the spectroscopic constants summarized in Table III of Ref. [57] (i.e., $B_{0}=$ $16488.3548 \mathrm{MHz}$ and $D_{0}=0.0312 \mathrm{MHz}$ ) to calculate the rotational levels of the AlF rotor in its electronic and vibrational
TABLE I. Summary of parameters used for the quantum coupled-channel calculations. Here, DR denotes the step size for integration, OTOL and DTOL the off-diagonal and diagonal crosssection tolerance thresholds for convergence, and NLEVEL the number of angular momentum quantum levels used to construct the basis set.

\begin{tabular}{lc}
\hline \hline$\mu$ (a.m.u.) & 3.68207364173 \\
\hline$B_{0}^{\mathrm{AlF}}\left(\mathrm{cm}^{-1}\right)$ & 0.5499923150168107 \\
$D_{j}^{\mathrm{AIF}}\left(\mathrm{cm}^{-1}\right)$ & 0.000001040719977 \\
$R_{\min }(\AA)$ & 1.6 \\
$R_{\max }(\AA)$ & 120 \\
DR $(\AA)$ & 0.008 \\
OTOL $\left(\AA^{2}\right)$ & 0.001 \\
DTOL $\left(\AA^{2}\right)$ & 0.1 \\
NLEVEL & 12 \\
\hline \hline
\end{tabular}

ground state. We used the MOLSCAT software package [73] to perform quantum multichannel scattering calculations of the rotationally inelastic cross sections based on the coupledchannel approach of Dalgarno et al. described in Sec. III. The coupled-channel equations were solved using the logderivative method of Manolopoulos [74] between 1.6 and $120 \AA$ with a step size of $0.008 \AA$. This method is an improved version of the Johnson's log-derivative algorithm [75], which propagates the log-derivative matrix $Y(R)=\Psi^{\prime}(R)[\Psi(R)]^{-1}$ instead of the wave function. The maximum total angular momentum of the system required to reach convergence is automatically decided by the code by setting the parameter JTOTU $^{1} \geqslant 999999$, and is found not to exceed a maximum value of 50 . In addition, all the multichannel calculations comprised 12 rotational states with $j=0-11$. A summary of the parameters used for the scattering calculations is given in Table I.

\section{RESULTS AND DISCUSSION}

The calculated quantum elastic and transport cross sections are shown in Fig. 3(a) as functions of collision energy. The cross sections are seen to exhibit three shape resonances, which occur at collision energies between 0.5 and $10 \mathrm{~K}$, which happens to be the energy range relevant for buffer-gas cooling experiments. At collision energies below $0.1 \mathrm{~K}$, one can see the emergence of the Wigner threshold behavior. This is consistent with the value of the van der Waals energy [16,28],

$$
E_{\mathrm{vdW}}=\left(\frac{2 \hbar^{6}}{\mu^{3} C_{6}^{0}}\right)^{1 / 2}
$$

which, for the $\mathrm{AlF}+\mathrm{He}$ system, evaluates to $E_{\mathrm{vdW}}=0.168 \mathrm{~K}$, where we made use of the $a b$ initio value of the $C_{6}^{0}$ pertaining to the spherical component $V_{0}$ of the potential, $C_{6}^{0}=-24.6$ a.u.

\footnotetext{
${ }^{1}$ JTOTU is a software specific keyword (Molscat) that refers to the upper limit of the total angular momentum required to achieve convergence based on a given value of the threshold. The built-in loop over JTOT (total angular momentum) runs from JTOTL to JTOTU.
} 

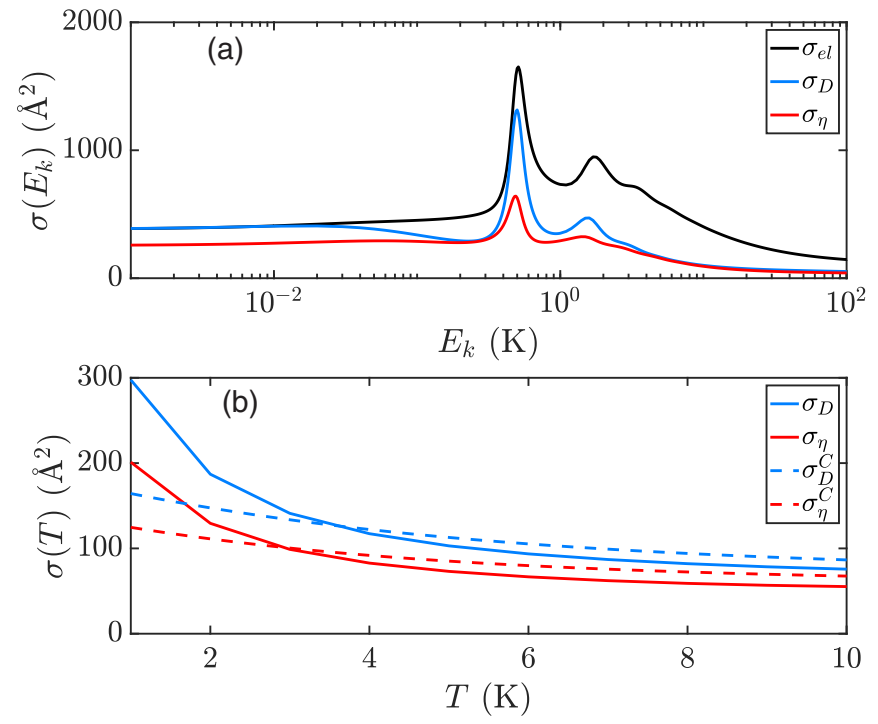

FIG. 3. Transport cross sections for AlF in cryogenic helium. (a) The elastic, diffusion, and viscosity cross sections as functions of the collision energy; cf. the $0 \rightarrow 0$ elastic cross section in Fig. 5 (thick blue). (b) The temperature-averaged quantum transport cross sections (full curves) on temperature. The corresponding classical cross sections are shown by dashed curves.

In the ultracold regime which, for the system considered, sets in below $100 \mathrm{mK}$, the diffusion cross section is seen to converge to the elastic one, whereas the viscosity cross section converges to a limit of its own. This behavior is due to the $s$-wave scattering that dominates the ultracold collision regime. For $s$-wave scattering, the phase shift is related to the scattering length $a$ via

$$
\lim _{k \rightarrow 0} \delta_{0}(k) \rightarrow-k a .
$$

By substituting this result into Eqs. (18)-(20), we obtain

$$
\begin{aligned}
& \lim _{k \rightarrow 0} \sigma_{D}\left(E_{k}\right) \rightarrow 4 \pi a^{2}, \\
& \lim _{k \rightarrow 0} \sigma_{\eta}\left(E_{k}\right) \rightarrow \frac{2}{3} 4 \pi a^{2}, \\
& \lim _{k \rightarrow 0} \sigma_{\mathrm{el}}\left(E_{k}\right) \rightarrow 4 \pi a^{2},
\end{aligned}
$$

which confirms the numerically found convergence behavior of the diffusion and viscosity cross sections displayed in Fig. 3(a).

A comparison between the classical and quantum temperature-averaged transport cross sections is shown in Fig. 3(b), which reveals that both treatments predict similar trends in the temperature dependence. For higher temperatures, the classical and quantum results are in a better agreement than at lower temperatures due to the washing out of contributions from a large number of partial waves. In the temperature range relevant for buffer-gas cooling experiments, i.e., $T \lesssim 10 \mathrm{~K}$, the discrepancy between the classical and quantum mechanical results becomes more significantand thus a quantum treatment of the transport cross sections is desirable.

We have also calculated the classical thermally averaged diffusion cross section at $20 \mathrm{~K}$ in order to compare it with

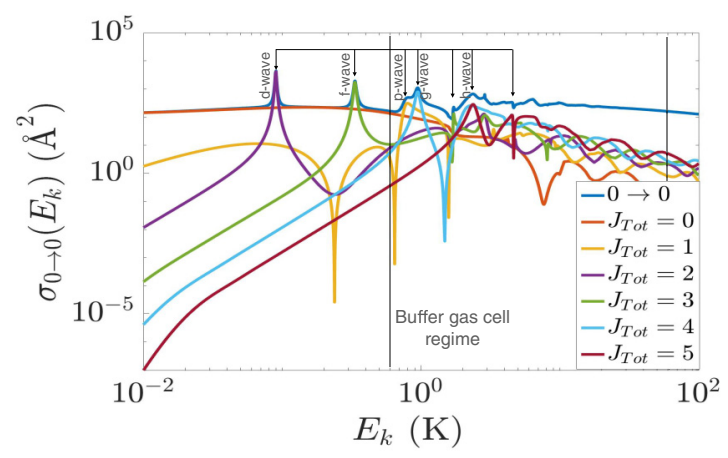

FIG. 4. Elastic cross sections and partial wave contributions. The $s-, p-, d-, f-, g-$ and $h$-wave partial contributions explain specific features of the elastic cross-section curve for the ground state $(j=0)$ of the AlF rigid rotor. The prominent shape resonances around $2.5 \mathrm{~K}$ occur in a buffer-gas cell experiment.

the previously reported results for $\operatorname{YbF}\left({ }^{2} \Sigma\right)$ in $\mathrm{He}$ [76] at that temperature. It turns out that the diffusion cross section of $\mathrm{AlF}$ in $\mathrm{He}$ is $70.13 \AA^{2}$, which comes quite close to the value of $79.6 \AA^{2}$ for $\mathrm{YbF}$ in He. This suggests that the transport cross sections for the two fluoride diatomic molecules could be independent of either molecular symmetry $\left({ }^{2} \Sigma\right.$ versus $\left.{ }^{1} \Sigma\right)$ or atomic properties ( $\mathrm{Yb}$ versus $\mathrm{Al}$ ). However, a more detailed study of other fluoride diatomic molecules is needed to assess the relevance of symmetry and atomic properties in order to come to a definite conclusion.

A molecule injected into a buffer-gas cell also undergoes relaxation of its internal degrees of freedom, in particular of molecular rotation. This process, termed rotational quenching, is driven by rotationally inelastic collisions from a given initial rotational state. In what follows, we present the results of our calculations of the rotationally inelastic $\mathrm{AlF}+\mathrm{He}$ collisions that are based on the multichannel coupled-channel approach described in Sec. III A.

The presence of inelastic channels affects the elastic cross section via the unitarity of the scattering matrix ([16], p. 26), leading to a cross-section value significantly different from that obtained in a single-channel calculation, as can be gleaned from Fig. 4. In particular, we find a greater number of resonances than in the elastic cross section; cf. Fig. 3(a). In addition, the resonances are often less distinct than in the single-channel elastic case because of their mutual proximity. These additional resonances come about for two main reasons: (1) the availability of more channels and (2) the anisotropy of the PES. Having more channels increases the probability of resonances, provided the underlying atommolecule interaction couples the channels. After performing a decomposition of the cross sections into partial waves, we were able to conclude that barring two resonances at 0.09 and $0.33 \mathrm{~K}$, the $s$-wave contribution dominates at collision energies of up to $0.67 \mathrm{~K}$. The remaining partial-wave contributions account for the first few prominent features of the elastic cross section. In particular, for collision energies $\gtrsim 10 \mathrm{~K}$, the different partial waves exhibit a rather oscillatory behavior that is averaged out once the partial waves are summed up to yield the elastic cross section. 


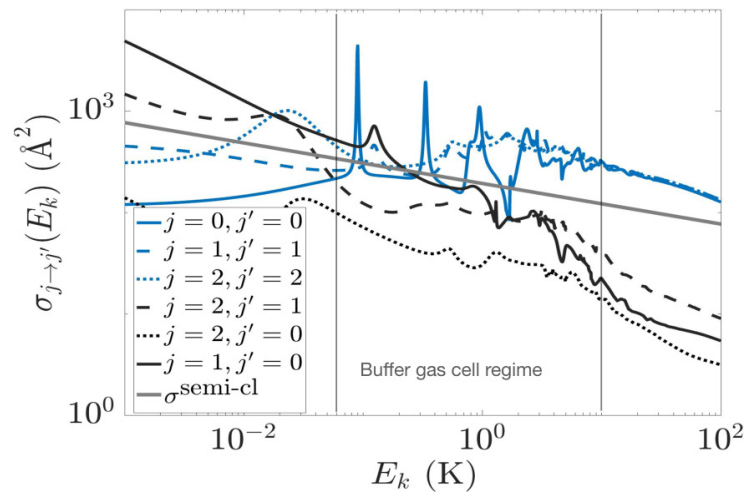

FIG. 5. Elastic and rotationally inelastic scattering cross sections for the first three initial rotational states $(j=0,1,2)$ of the AlF rotor. For collision energies greater than about $1.7 \mathrm{~K}$, elastic scattering begins to dominate over all inelastic scattering channels. Note also the thick gray curve representing the semiclassical elastic scattering cross section; see text.

In Fig. 5, we show both elastic and inelastic cross sections for the first three rotational states of $\operatorname{AlF}(j=0,1,2)$ as functions of collision energy. At low collision energies (the left-most regime), quantum behavior dominates the dynamics, leading to a pronounced state-dependent effect on the elastic and inelastic cross sections. For instance, the elastic cross section tends to a different scattering length depending on the rotational state considered. As a point of reference, we add the semiclassical elastic cross section to the plot (thick gray curve), given by

$$
\sigma_{\mathrm{el}}^{\text {semicl }}=\left(\frac{6 \pi^{3} C_{6}^{0} \Gamma[7 / 2]}{h v_{\mathrm{coll}} \Gamma[4]}\right)^{2 / 5}
$$

with $v_{\text {coll }}=\left(2 E_{\text {coll }} / \mu\right)^{1 / 2}$. The positions of the resonances in both the elastic and inelastic cross sections depend significantly on the initial and final rotational states. The rotational-state dependence of the inelastic cross sections follows in part from energetic considerations (the larger the energy gap between different initial and final states, the lower the transition probability). However, additional features of the system in question need to be included in order to rationalize its behavior: For instance, the inelastic cross section $\sigma_{2 \rightarrow 0}\left(E_{k}\right)$ is less than $\sigma_{1 \rightarrow 0}\left(E_{k}\right)$ because the two processes are driven, respectively, by the $V_{2}(R)$ and $V_{1}(R)$ components of the potential and $\left|V_{2}(R)\right|<\left|V_{1}(R)\right|$, as revealed by our ab initio PES calculations.

The transport rate constants of a fluid [72] can be obtained from the transport cross sections introduced in Sec. III B. In the absence of molecular vibration, the rotational state-to-state rate constants are given by [80]

$$
\begin{aligned}
k_{j \rightarrow j^{\prime}}(T) & =\left\langle\sigma_{j \rightarrow j^{\prime}}\left(E_{k}\right) v\right\rangle \\
& =\left(\frac{8}{\pi \mu \beta^{3}}\right)^{1 / 2} \int_{0}^{\infty} \sigma_{j \rightarrow j^{\prime}}\left(E_{k}\right) \exp \left(-E_{k} / \beta\right) E_{k} d E_{k},
\end{aligned}
$$

with $\beta=k_{B} T$. The dependence of the state-to-state rate constants on temperature is displayed in Fig. 6 for a temperature range relevant to buffer-gas cooling. What we see is a large

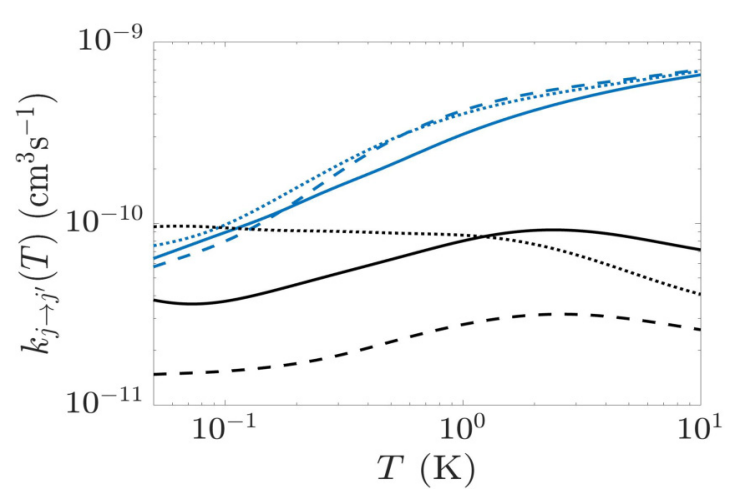

FIG. 6. Rate constants for elastic (blue curves) and rotationally inelastic (black curves) processes as functions of temperature. The elastic rates dominate over the inelastic ones beyond collision energies in excess of $0.1 \mathrm{~K}$. For a detailed legend, see Fig. 5.

difference between the rates of elastic and inelastic scattering in the cryogenic buffer gas. Similarly as in the case of the cross sections, the rate constants for the exchange of the largest rotational quanta are suppressed. However, the elastic rate constants are almost independent of the rotational state, which reflects the average nature of the rate coefficient.

Figure 6 also reveals that the state-to-state rate constants corresponding to a relaxation of the rotational degrees of freedom of the molecule are only weakly temperature dependent. A similar behavior has been reported for other ${ }^{1} \Sigma-\mathrm{He}$ collision systems, as summarized in Table II. The more efficient relaxation of AlF in $\mathrm{He}$ can be rationalized by invoking the adiabaticity parameter, $\xi=\tau_{\text {coll }} / \tau_{\text {rot }} \propto B_{0} / \sqrt{\mu}$, where $\tau_{\text {coll }}$ and $\tau_{\text {rot }}$ are, respectively, the collision time and rotational period of the molecule; see, also, Table I. For $\xi \gg 1$, the collision time exceeds the rotational period and hence the collision proceeds adiabatically, without changing the rotational state of the molecule. On the other hand, for $\xi \ll 1$, the collision process is nonadiabatic, leading to efficient rotational energy transfer. Therefore, heavier molecules with smaller rotational constants, such as AlF compared with $\mathrm{CO}$ or HF, lead to small values of $\xi$ and thus a more nonadiabatic behavior (more facile rotational quenching). Based on the same argument, we conclude that the MgF-He system will show relaxation rates similar to those of the AlF-He system, but that BaF-He (also see Ref. [81]) will exhibit a more efficient quenching due to BaF's smaller rotational constant [65].

Given that the inaccuracy of the ab initio quantum chemistry calculations exceeds the collision energies of $\lesssim 100 \mathrm{~K}$

TABLE II. State-to-state rate constants $k_{1 \rightarrow 0}(T)$ in $\mathrm{cm}^{3} \mathrm{~s}^{-1}$ for different ${ }^{1} \Sigma^{+}$-He scattering systems. The values of the constants for the HF-He have been taken from Ref. [77], CO-He from Ref. [78], and ThO-He from Ref. [79].

\begin{tabular}{lccc}
\hline \hline System & $T=4 \mathrm{~K}$ & $T=5 \mathrm{~K}$ & $T=10 \mathrm{~K}$ \\
\hline HF-He & $5 \times 10^{-12}$ & $5 \times 10^{-12}$ & $5 \times 10^{-12}$ \\
CO-He & & $3.4 \times 10^{-11}$ & $3.2 \times 10^{-11}$ \\
ThO-He & $3 \times 10^{-11}$ & & \\
AlF-He & $8.79 \times 10^{-11}$ & $8.4 \times 10^{-11}$ & $7.1 \times 10^{-11}$ \\
\hline \hline
\end{tabular}




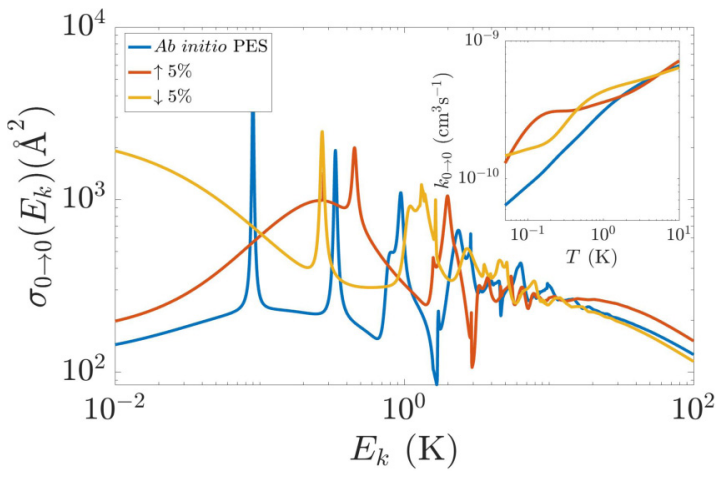

FIG. 7. Sensitivity to shifts in the PES up $(\uparrow)$ or down $(\downarrow)$ by $5 \%$. The density of resonances essentially remains unchanged within the collision energy regime accessible to buffer-gas experiments. The inset shows rate coefficients for rotational quenching as a function of temperature.

that are at play in cryogenic helium cooling, we tested how sensitive the elastic scattering cross sections are to changes of the PES. To this end, the PES was either raised or lowered throughout by $5 \%$ and the calculations repeated. The error thus simulated overestimates the inaccuracies of our method in the long-range tail of the potential, but may underestimate them in the short-range region. The simulations are displayed in Fig. 7, which attests how the positions and the widths of the resonances are affected. Nevertheless, the density of the resonances remains unchanged. The simulation also reveals that in the thermal regime, for energies greater than $10 \mathrm{~K}$, the cross sections are less sensitive to changes in the PES, as expected. The inset of Fig. 7 shows the effects of varying the PES on the state-to-state rate constants, which are seen to remain qualitatively unaffected.

Finally, we offer a simple estimate of the time taken to thermalize AlF molecules in a helium buffer gas via a simple model [82] based on elastic collisions between two hard spheres. Consider the following differential equation:

$$
\frac{d T}{d t}=-R\left(T-T_{\mathrm{bg}}\right) / \kappa,
$$

where $R$ is the collision rate, $T$ is the temperature of the species being cooled, $T_{\mathrm{bg}}$ is the buffer-gas temperature, and $\kappa=\left(m_{\mathrm{AlF}}+m_{\mathrm{He}}\right)^{2} /\left(2 m_{\mathrm{AlF}} m_{\mathrm{He}}\right)$. The collision rate is the product of the density of the helium atoms, collision cross section, and the averaged relative speed (which is nearly the same as the speed of the helium atoms). Assuming that the starting temperature of the AlF molecule, after ablation of $\mathrm{Al}$ and reaction with a fluoride donor gas, is around $3000 \mathrm{~K}$, and a helium density of $10^{15} \mathrm{~cm}^{-3}$ with an average helium atom speed of $120 \mathrm{~m} / \mathrm{s}$, we arrive at a thermalization time (i.e., time taken for the AlF molecule to fall within about $30 \%$ of the helium buffer temperature) of $0.13 \mathrm{~ms}$ in a helium buffer gas at $4 \mathrm{~K}$, requiring about 55 collisions. The thermalization timescale is expected to be reduced by a factor of two for a buffer gas at around $2 \mathrm{~K}$, depending on the exact location of the broad shape resonance, the cross-section resonance that could be higher than the one at $4 \mathrm{~K}$ by as much as a factor of three.

\section{CONCLUSIONS}

We undertook a study of the collisions between aluminum monofluoride (AlF) molecules and helium (He) atoms at collision energies relevant for buffer-gas cooling by cryogenic helium. Our study, based on an accurate $a b$ initio potential energy surface and quantum multichannel scattering theory, revealed high thermalization rates for both translational and rotational degrees of freedom of the molecule, on the order of $10^{10}$ and $10^{11} \mathrm{~cm}^{3} \mathrm{~s}^{-1}$, respectively.

The large anisotropy of the AlF-He PES (due to the preferred $\mathrm{He}-\mathrm{F}$ attraction over that between $\mathrm{He}$ and $\mathrm{Al}$ ) and a small rotational constant of AlF translates into a complex resonance structure in elastic and rotationally inelastic scattering cross sections. However, at collision energies $\gtrsim 10 \mathrm{~K}$, the quantum effects are found to average out and most of the scattering observables become independent of the rotational state of the molecule. Classical calculations of the transport cross sections are found to be valid up to temperatures of $5 \mathrm{~K}$ with a relative error $\lesssim 15 \%$.

Although possible inaccuracies of the PES may affect the positions of the resonances, their density was found to remain the same. Moreover, we showed that the state-to-state rate constants are quite robust against inaccuracies of the PES for temperatures relevant to buffer-gas cell experiments. These findings confirm AlF as a suitable candidate for efficient sympathetic cooling.

Our results on the AlF-He system are of a more general interest. For one, our classical transport cross sections benchmark the accuracy of such methods for ${ }^{1} \Sigma$ molecule-He collisions. Furthermore, considering the adiabaticity parameter, we estimate that $\mathrm{MgF}$ will be quenched as efficiently as AlF. Finally, owing to its smaller rotational constant, we expect $\mathrm{BaF}$ to be quenched more efficiently than AlF.

\section{ACKNOWLEDGMENTS}

M.K. acknowledges support by the International Max Planck Research School for Elementary Processes in Physical Chemistry (IMPRS-EPPC). B.F. thanks John Doyle and Hossein Sadeghpour for their hospitality during his stay at the Harvard Department of Physics and at the HarvardSmithsonian Institute for Theoretical Atomic, Molecular, and Optical Physics (ITAMP).
[1] M. S. Safronova, D. Budker, D. DeMille, Derek F. Jackson Kimball, A. Derevianko, and C. W. Clark, Search for new physics with atoms and molecules, Rev. Mod. Phys. 90, 025008 (2018).
[2] J. Mur-Petit, J. J. García-Ripoll, J. Pérez-Ríos, J. CamposMartínez, M. I. Hernández, and S. Willitsch, Temperatureindependent quantum logic for molecular spectroscopy, Phys. Rev. A 85, 022308 (2012). 
[3] M. Shi, P. F. Herskind, M. Drewsen, and I. L. Chuang, Microwave quantum logic spectroscopy and control of molecular ions, New J. Phys. 15, 113019 (2013).

[4] F. Wolf, Y. Wan, J. C. Heip, F. Gebert, C. Shi, and P. O. Schmidt, Non-destructive state detection for quantum logic spectroscopy of molecular ions, Nature (London) 530, 457 (2016).

[5] C.-w. Chou, C. Kurz, D. B. Hume, P. N. Plessow, D. R. Leibrandt, and D. Leibfried, Preparation and coherent manipulation of pure quantum states of a single molecular ion, Nature (London) 545, 203 (2017).

[6] C. W. Chou, A. L. Collopy, C. Kurz, Y. Lin, M. E. Harding, P. N. Plessow, T. Fortier, S. Diddams, D. Leibfried, and D. R. Leibrandt, Frequency-comb spectroscopy on pure quantum states of a single molecular ion, Science 367, 1458 (2020).

[7] P. Puri, S. J. Schowalter, S. Kotochigova, A. Petrov, and E. R. Hudson, Action spectroscopy of srcl+ using an integrated ion trap time-of-flight mass spectrometer, J. Chem. Phys. 141, 014309 (2014).

[8] S. Brünken, L. Kluge, A. Stoffels, J. Pérez-Ríos, and S. Schlemmer, Rotational state-dependent attachment of He atoms to cold molecular ions: An action spectroscopic scheme for rotational spectroscopy, J. Mol. Spectrosc. 332, 67 (2017).

[9] B. A. McGuire, O. Asvany, S. Brünken, and S. Schlemmer, Laboratory spectroscopy techniques to enable observations of interstellar ion chemistry, Nat. Rev. Phys. 2, 402 (2020).

[10] J. Doyle, B. Friedrich, R. V. Krems, and F. Masnou-Seeuws, Editorial: Quo vadis, cold molecules? Europhys. J. D 31, 149 (2004).

[11] R. V. Krems, Cold controlled chemistry, Phys. Chem. Chem. Phys. 10, 4079 (2008).

[12] L. D. Carr, D. DeMille, R. V. Krems, and J. Ye, Cold and ultracold molecules: Science, technology, and applications, New J. Phys. 11, 055049 (2009).

[13] M. Schnell and G. Meijer, Cold molecules: Preparation, applications, and challenges, Angew. Chem., Intl. Ed. 48, 6010 (2009).

[14] G. Quéméner and P. S. Julienne, Ultracold molecules under control! Chem. Rev. 112, 4949 (2012).

[15] N. Balakrishnan, Perspective: Ultracold molecules and the dawn of cold controlled chemistry, J. Chem. Phys. 145, 150901 (2016).

[16] J. P. Ríos, An Introduction to Cold and Ultracold Chemistry (Springer, Cham, Switzerland, 2020).

[17] D. DeMille, Quantum Computation with Trapped Polar Molecules, Phys. Rev. Lett. 88, 067901 (2002).

[18] M. Karra, K. Sharma, B. Friedrich, S. Kais, and D. Herschbach, Prospects for quantum computing with an array of ultracold polar paramagnetic molecules, J. Chem. Phys. 144, 094301 (2016).

[19] P. Yu, L. W. Cheuk, I. Kozyryev, and J. M. Doyle, A scalable quantum computing platform using symmetric-top molecules, New J. Phys. 21, 093049 (2019).

[20] K. W. Madison, Y. Wang, A. M. Rey, and K. Bongs, Annual Review of Cold Atoms and Molecules (World Scientific, Singapore, 2013).

[21] J. A. Blackmore, L. Caldwell, P. D. Gregory, E. M. Bridge, R. Sawant, J. Aldegunde, J. Mur-Petit, D. Jaksch, J. M. Hutson, B. E. Sauer, M. R. Tarbutt, and S. L. Cornish, Ultracold molecules for quantum simulation: Rotational coherences in CaF and RbCs, Quantum Sci. Technol. 4, 014010 (2018).
[22] J. Zhu, S. Kais, Q. Wei, D. Herschbach, and B. Friedrich, Implementation of quantum logic gates using polar molecules in pendular states, J. Chem. Phys. 138, 024104 (2013).

[23] R. Sawant, J. A. Blackmore, P. D. Gregory, J. Mur-Petit, D. Jaksch, J. Aldegunde, J. M. Hutson, M. R. Tarbutt, and S. L. Cornish, Ultracold polar molecules as qudits, New J. Phys. 22, 013027 (2020).

[24] A. Micheli, G. K. Brennen, and P. Zoller, A toolbox for latticespin models with polar molecules, Nat. Phys. 2, 341 (2006).

[25] F. Herrera and R. V. Krems, Tunable Holstein model with cold polar molecules, Phys. Rev. A 84, 051401(R) (2011).

[26] A. Fioretti, D. Comparat, A. Crubellier, O. Dulieu, F. MasnouSeeuws, and P. Pillet, Formation of Cold $\mathrm{Cs}_{2}$ Molecules Through Photoassociation, Phys. Rev. Lett. 80, 4402 (1998).

[27] J. L. Bohn and P. S. Julienne, Semianalytic theory of laserassisted resonant cold collisions, Phys. Rev. A 60, 414 (1999).

[28] K. M. Jones, E. Tiesinga, P. D. Lett, and P. S. Julienne, Ultracold photoassociation spectroscopy: Long-range molecules and atomic scattering, Rev. Mod. Phys. 78, 483 (2006).

[29] J. Pérez-Ríos, M. Lepers, and O. Dulieu, Theory of Long-Range Ultracold Atom-Molecule Photoassociation, Phys. Rev. Lett. 115, 073201 (2015).

[30] D. B. Blasing, I. C. Stevenson, J. Pérez-Ríos, D. S. Elliott, and Y. P. Chen, Short-range photoassociation of LiRb, Phys. Rev. A 94, 062504 (2016).

[31] T. Köhler, K. Góral, and P. S. Julienne, Production of cold molecules via magnetically tunable Feshbach resonances, Rev. Mod. Phys. 78, 1311 (2006).

[32] C. Chin, R. Grimm, P. Julienne, and E. Tiesinga, Feshbach resonances in ultracold gases, Rev. Mod. Phys. 82, 1225 (2010).

[33] K.-K. Ni, S. Ospelkaus, M. H. G. de Miranda, A. Pe'er, B. Neyenhuis, J. J. Zirbel, S. Kotochigova, P. S. Julienne, D. S. Jin, and J. Ye, A high phase-space-density gas of polar molecules, Science 322, 231 (2008)

[34] J. G. Danzl, E. Haller, M. Gustavsson, M. J. Mark, R. Hart, N. Bouloufa, O. Dulieu, H. Ritsch, and H.-C. Nägerl, Quantum gas of deeply bound ground state molecules, Science 321, 1062 (2008).

[35] X. Ye, M. Guo, M. L. González-Martínez, G. Quéméner, and D. Wang, Collisions of ultracold ${ }^{23} \mathrm{Na}^{87} \mathrm{Rb}$ molecules with controlled chemical reactivities, Sci. Adv. 4, eaaq0083 (2018).

[36] L. De Marco, G. Valtolina, K. Matsuda, W. G. Tobias, J. P. Covey, and J. Ye, A degenerate fermi gas of polar molecules, Science 363, 853 (2019).

[37] H. Yang, D.-C. Zhang, L. Liu, Y.-X. Liu, J. Nan, B. Zhao, and J.-W. Pan, Observation of magnetically tunable Feshbach resonances in ultracold ${ }^{23} \mathrm{Na}^{40} \mathrm{~K}+{ }^{40} \mathrm{~K}$ collisions, Science 363, 261 (2019).

[38] K. K. Voges, P. Gersema, M. Meyer zum Alten Borgloh, T. A. Schulze, T. Hartmann, A. Zenesini, and S. Ospelkaus, Ultracold Gas of Bosonic ${ }^{23} \mathrm{Na}^{39} \mathrm{~K}$ Ground-State Molecules, Phys. Rev. Lett. 125, 083401 (2020).

[39] M. Mayle, B. P. Ruzic, and J. L. Bohn, Statistical aspects of ultracold resonant scattering, Phys. Rev. A 85, 062712 (2012).

[40] A. Christianen, M. W. Zwierlein, G. C. Groenenboom, and T. Karman, Photoinduced Two-Body Loss of Ultracold Molecules, Phys. Rev. Lett. 123, 123402 (2019).

[41] P. D. Gregory, M. D. Frye, J. A. Blackmore, E. M. Bridge, R. Sawant, J. M. Hutson, and S. L. Cornish, Sticky collisions of ultracold RbCs molecules, Nat. Commun. 10, 3104 (2019). 
[42] J. D. Weinstein, R. deCarvalho, T. Guillet, B. Friedrich, and J. M. Doyle, Magnetic trapping of calcium monohydride molecules at millikelvin temperatures, Nature (London) 395, 148 (1998).

[43] H. L. Bethlem, G. Berden, and G. Meijer, Decelerating Neutral Dipolar Molecules, Phys. Rev. Lett. 83, 1558 (1999).

[44] N. Vanhaecke, U. Meier, M. Andrist, B. H. Meier, and F. Merkt, Multistage Zeeman deceleration of hydrogen atoms, Phys. Rev. A 75, 031402(R) (2007).

[45] E. Narevicius, A. Libson, C. G. Parthey, I. Chavez, J. Narevicius, U. Even, and M. G. Raizen, Stopping supersonic oxygen with a series of pulsed electromagnetic coils: A molecular coilgun, Phys. Rev. A 77, 051401(R) (2008).

[46] M. Zeppenfeld, B. G. U. Englert, R. Glöckner, A. Prehn, M. Mielenz, C. Sommer, L. D. van Buuren, M. Motsch, and G. Rempe, Sisyphus cooling of electrically trapped polyatomic molecules, Nature (London) 491, 570 (2012).

[47] E. S. Shuman, J. F. Barry, and D. DeMille, Laser cooling of a diatomic molecule, Nature (London) 467, 820 (2010).

[48] M. R. Tarbutt, Laser cooling of molecules, Contemp. Phys. 59, 356 (2018).

[49] E. Hudson, Laser cooling of larger quantum objects, Science 369, 1304 (2020).

[50] D. Mitra, N. B. Vilas, C. Hallas, L. Anderegg, B. L. Augenbraun, L. Baum, C. Miller, S. Raval, and J. M. Doyle, Direct laser cooling of a symmetric top molecules, Science $\mathbf{3 6 9}$, 1366 (2020).

[51] B. L. Augenbraun, Z. D. Lasner, A. Frenett, H. Sawaoka, C. Miller, T. C. Steimle, and J. M. Doyle, Laser-cooled polyatomic molecules for improved electron electric dipole moment searches, New J. Phys. 22, 022003 (2020).

[52] B. L. Augenbraun, J. M. Doyle, T. Zelevinsky, and I. Kozyryev, Molecular Asymmetry and Optical Cycling: Laser Cooling Asymmetric Top Molecules, Phys. Rev. X 10, 031022 (2020).

[53] M. D. Di Rosa, Laser-cooling molecules, Europhys. J. D 31, 395 (2004).

[54] M. V. Ivanov, F. H. Bangerter, and A. I. Krylov, Towards a rational design of laser-coolable molecules: Insights from equation-of-motion coupled-cluster calculations, Phys. Chem. Chem. Phys. 21, 19447 (2019).

[55] M. V. Ivanov, T.-C. Jagau, G.-Z. Zhu, E. R. Hudson, and A. I. Krylov, In search of molecular ions for optical cycling: A difficult road, PhysChemChemPhys 22, 17075 (2020).

[56] J. Kłos and S. Kotochigova, Prospects for laser cooling of polyatomic molecules with increasing complexity, Phys. Rev. Res. 2, 013384 (2020).

[57] S. Truppe, S. Marx, S. Kray, M. Doppelbauer, S. Hofsäss, H. C. Schewe, N. Walter, J. Pérez-Riós, B. G. Sartakov, and G. Meijer, Spectroscopic characterization of aluminum monofluoride with relevance to laser cooling and trapping, Phys. Rev. A 100, 052513 (2019).

[58] S. Hofsäss, M. Doppelbauer, S. Wright, S. Kray, B. Sartakov, J. Pérez-Rós, G. Meijer, and S. Truppe, Optical cycling of AlF molecules, New J. Phys. 23075001 (2021).

[59] M. Doppelbauer, N. Walter, S. Hofsäss, S. Marx, H. C. Schewe, S. Kray, J. Pérez-Ríos, B. G. Sartakov, S. Truppe, and G. Meijer, Characterization of the state and its interaction with the state in aluminium monofluoride, Mol. Phys. 119, e1810351 (2020).
[60] J. L. Highberger, C. Savage, J. H. Bieging, and L. M. Ziurys, Heavy-metal chemistry in proto-planetary nebulae: Detection of MgNC, $\mathrm{NaCN}$, and $\mathrm{AlF}$ toward CRL 2688, Astrophys. J. 562, 790 (2001).

[61] T. Kamiński, R. Tylenda, K. M. Menten, A. Karakas, J. M. Winters, A. A. Breier, K. T. Wong, T. F. Giesen, and N. A. Patel, Astronomical detection of radioactive molecule 26AlF in the remnant of an ancient explosion, Nat. Astron. 2, 778 (2018).

[62] R. Diehl, H. Halloin, K. Kretschmer, G. G. Lichti, V. Schönfelder, A. W. Strong, A. v. Kienlin, W. Wang, P. Jean, J. Knödlseder, J.-P. Roques, G. Weidenspointner, S. Schanne, D. H. Hartmann, C. Winkler, and C. Wunderer, Radioactive ${ }^{2} 6 \mathrm{Al}$ from massive stars in the Galaxy, Nature (London) 439, 45 (2006).

[63] H.-J. Werner, P. J. Knowles, G. Knizia, F. R. Manby, M. Schütz, P. Celani, W. Györffy, D. Kats, T. Korona, R. Lindh, A. Mitrushenkov, G. Rauhut, K. R. Shamasundar, T. B. Adler, R. D. Amos, S. J. Bennie, A. Bernhardsson, A. Berning, D. L. Cooper, M. J. O. Deegan et al., and MOLPRO, version 2019.2, a package of ab initio programs (Stuttgart, Germany, 2019).

[64] K. L. Schuchardt, B. T. Didier, T. Elsethagen, L. Sun, V. Gurumoorthi, J. Chase, J. Li, and T. L. Windus, Basis set exchange: A community database for computational sciences, J. Chem. Inf. Mod. 47, 1045 (2007).

[65] X. Liu, S. Truppe, G. Meijer, and J. Pérez-Ríos, The diatomic molecular spectroscopy database, J. Chem. 12, 31 (2020).

[66] T. V. Tscherbul, J. Kłos, L. Rajchel, and R. V. Krems, Fine and hyperfine interactions in cold ybf-He collisions in electromagnetic fields, Phys. Rev. A 75, 033416 (2007).

[67] N. Gotoum, C. Nkem, K. Hammami, M. Ahamat Charfadine, L. C. Owono Owono, and N.-E. Jaidane, Rotational excitation of aluminium monofluoride (AlF) by $\mathrm{He}$ atom at low temperature, Astrophys. Space Sci. 332, 209 (2011).

[68] A. M. Arthurs, A. Dalgarno, and D. R. Bates, The theory of scattering by a rigid rotator, Proc. R. Soc. London A 256, 540 (1960).

[69] A fragment represents the distinct energy levels of the system under consideration in the asymptotic region. Therefore, a fragment corresponds to many channels in the interaction region.

[70] N. F. Mott and S. W. Massey, The Theory of Atomic Collisions (Oxford University Press, Oxford, 1965).

[71] E. Mason and T. Marrero, The Diffusion of Atoms and Molecules (Academic Press, Washington, D.C., 1970), pp. 155-232.

[72] J. O. Hirschfelder, C. F. Curtiss, and R. B. Bird, Molecular Theory of Gases and Liquids (Wiley, New York, 1964).

[73] J. M. Hutson and C. R. Le Sueur, Molscat: A program for nonreactive quantum scattering calculations on atomic and molecular collisions, Comput. Phys. Commun. 241, 9 (2019).

[74] D. E. Manolopoulos, An improved log derivative method for inelastic scattering, J. Chem. Phys. 85, 6425 (1986).

[75] B. Johnson, The multichannel log-derivative method for scattering calculations, J. Comput. Phys. 13, 445 (1973).

[76] S. M. Skoff, R. J. Hendricks, C. D. J. Sinclair, J. J. Hudson, D. M. Segal, B. E. Sauer, E. A. Hinds, and M. R. Tarbutt, Diffusion, thermalization, and optical pumping of Ybf molecules in a cold buffer-gas cell, Phys. Rev. A 83, 023418 (2011).

[77] B. Yang, K. M. Walker, R. C. Forrey, P. C. Stancil, and N. Balakrishnan, Collisional quenching of highly rotationally excited HF, A\&A 578, A65 (2015). 
[78] C. Cecchi-Pestellini, E. Bodo, N. Balakrishnan, and A. Dalgarno, Rotational and vibrational excitation of $\mathrm{CO}$ molecules by collisions with $4 \mathrm{He}$ atoms, Astrophys. J. 571, 1015 (2002).

[79] T. V. Tscherbul, E. R. Sayfutyarova, A. A. Buchachenko, and A. Dalgarno, $\mathrm{He}-\mathrm{ThO}\left({ }^{1} \Sigma^{+}\right)$interactions at low temperatures: Elastic and inelastic collisions, transport properties, and complex formation in cold 4He gas, J. Chem. Phys. 134, 144301 (2011).

[80] S. Montero and J. Pérez-Ríos, Rotational relaxation in molecular hydrogen and deuterium: Theory versus acoustic experiments, J. Chem. Phys. 141, 114301 (2014).

[81] R. Albrecht, M. Scharwaechter, T. Sixt, L. Hofer, and T. Langen, Buffer-gas cooling, high-resolution spectroscopy, and optical cycling of barium monofluoride molecules, Phys. Rev. A 101, 013413 (2020).

[82] R. deCarvalho, J. M. Doyle, B. Friedrich, T. Guillet, J. Kim, D. Patterson, and J. D. Weinstein, Buffer-gas loaded magnetic traps for atoms and molecules: A primer, Europhys. J. D 7, 289 (1999). 